\title{
Pathological Journeys or Help Seeking Behavior: The Case of Travelers to Geneva, An International City
}

\author{
Ariel Eytan", Sophie Favre, Marianne Gex-Fabry, Laurence Borras, François Ferrero and \\ Gilles Bertschy
}

\begin{abstract}
Division of Adult Psychiatry, Department of Psychiatry, University Hospitals of Geneva, 2, Chemin du Petit-Bel-Air, CH-1225 Geneva, Switzerland
\end{abstract}

\begin{abstract}
Several international organizations have their headquarters in Geneva and are thus hypothesized to attract both people seeking help for human right abuses and also pathological travelers. The present study aimed at characterizing pathological travelers, a population seldom studied in psychiatry. Patients referred for pathological journeys to the only psychiatric hospital in Geneva were retrospectively identified on the basis of medical certificates. Their medical records were then analyzed. The study covered a 4 -year period. Seventy-six patients were identified. They were in majority male, aged less than 50 and single; $62 \%$ came from European countries and $84 \%$ had some prior psychiatric history; $58 \%$ were diagnosed with a psychotic disorder, mainly schizophrenia, acute transient psychotic disorder and delusional disorder. Sixteen patients $(21 \%)$ had been attracted to Geneva specifically because of its international organizations, while 60 came for other reasons. Prevalence of persecutory delusions was significantly higher among travelers attracted by the "international Geneva" ( $88 \%$ versus $43 \%, \mathrm{P}<0.005)$. Pathological journeys represent an identifiable cause of psychiatric hospitalization in Geneva. Prospective studies should be conducted using a more precise and restrictive definition of this entity in order to avoid stigmatization of people crossing borders for other motives.
\end{abstract}

\section{INTRODUCTION}

Several medical terms encompass the notion of pathological journey. Impulsive journey, compulsive travel, fugue states, ambulatory delirium, paranoid flight and wandering have all been used to describe patients who travel in unprepared or precarious ways in relationship with a psychiatric condition [1]. The more pejorative expression "psychiatric tourism" is also mentioned by some authors [2]. However, despite quite abundant clinical descriptions and vocabulary, very few studies deal expressly with this type of behavior.

One of the main reasons for this scarcity of data in this particular field is that pathological journey does not exist as a single diagnostic category amongst the main psychiatric classifications. In DSM IV [3], the topic is discussed in the diagnostic category of fugues, which occur during dissociation states. These fugues are sudden occurrences, accompanied by amnesia and confusion affecting personal identity. The differential diagnosis section mentions the fact that fugues or wandering may occur in general medical conditions, as well as during epileptic seizures or substance abuse disorders. Two other important mental disorders should be considered: purposeful travel can occur during manic episodes and peripatetic behavior is sometimes observed in cases of schizophrenia or delusional disorders. The categorization of fugues is similar in ICD-10 [4]. Pathological journey is originally a French concept which refers to episodes of mental illness in which travel plays a key part [5].

According to available data, pathological journeys are not exceptional, especially in large cities. Tannock and

*Address correspondence to this author at the Department of Psychiatry, Division of Adult Psychiatry, HUG, 2 Chemin du Petit-Bel-Air, bâtiment Voirons, 1225 Geneva, Switzerland; Tel: +4122 30547 55;

E-mail: Ariel.Eytan@hcuge.ch
Turner, discussing the overloading of London psychiatric beds, took the following example: "Over the past two weeks one 17-bed ward in Hackney has admitted three nonLondoners: a psychotic patient from Sierra Leone, a Barbadian with learning difficulties, and a manic Australian. On a similar ward last month there were five non-Londoners: two manic patients (from New York and Argentina), a mute man from Guadeloupe, a Nigerian with persecutory delusions, and a Kurdish patient seeking asylum who had been in Britain for two months. Currently, on one ward five patients are of no fixed abode" [2].

Controversy exists regarding pathological journeys to specific places. Authors in Israel have argued that there in a genuine "Jerusalem syndrome", afflicting ardent religious believers who go to Jerusalem and develop psychotic symptoms during their stay [6]. It is not clear however if this syndrome should be regarded as a unique cultural phenomenon due to its overwhelming theatrical characteristics [7], or as the aggravation of an underlying psychotic disorder $[8,9]$. To a lesser degree, a similar debate took place regarding possible specificities of psychotic episodes occurring among travelers to India [10]. Anecdotic reports of Paris and New York syndromes (affecting Japanese tourists disappointed by the city or confronted with situations of violence) and of Stendhal's syndrome (affecting travelers to cities of art and culture, such as that described by Stendhal in 1817 after a visit to Florence, Italy) may be found in the media.

Our clinical practice indicates that psychiatric hospitalizations for pathological travels represent a significant phenomenon in Geneva, Switzerland. Some of these travelers appear not to have chosen this city randomly, but precisely because of its international reputation. They are usually admitted to the hospital following inappropriate visits to one of the many International Organizations with headquarters in Geneva, such as the United Nations (UN) and its different 
agencies. Our study was designed to assess this clinical phenomenon.

\section{METHODS}

\section{Patients}

We conducted a retrospective, case register study covering a 4-year period (November 2000 to November 2004). It included patients hospitalized at the only psychiatric hospital in Geneva, Switzerland (providing for a catchment area of approximately 430'000 inhabitants). The list of all hospital admissions was examined. When medical admission certificates bore the mention of pathological journey, the medical record was obtained and analyzed. Variables of interest concerned usual socio-demographic factors, diagnosis (our hospital diagnoses at discharge are ICD-10 diagnoses), psychiatric antecedents, country of origin, variables related to travel (alone or not, possible connections in Geneva) and characteristics of hospitalization (manner and motives of admission, duration of stay, psychotropic treatment, destination at discharge). The study protocol was approved by the ethics committee of the Psychiatry Department, Geneva University Hospitals.

\section{Statistics}

Group comparisons were performed with the Fisher's exact test for categorical variables and the Student's t-test for continuous variables. Significance level was set at 0.05 (twosided tests). Statistical analysis was performed with SPSS version 11, with the exact tests option installed (SPSS Inc., Chicago, IL).

\section{RESULTS}

A total number of $11^{\prime} 950$ hospitalizations were recorded during the 4-year period. Among those hospital admissions, less than $1 \%(\mathrm{~N}=104)$ were associated with a pathological journey mention on admission records. Twenty-eight cases were excluded as they concerned Swiss patients repatriated from abroad.

The present study thus included 76 admissions, corresponding to a sample of 76 different patients. Sociodemographic and medical characteristics are presented in Table 1. A majority of patients were male (58\%), aged less than $50(75 \%)$ and single $(66 \%)$. In $62 \%$ of cases, they came from a European country (mainly France, Italy, Germany and Russia). Prior psychiatric history was documented for $84 \%$ of patients, and discontinued treatment for $63 \%$. Most of patients traveled alone (92\%), came directly from their home country to Geneva $(62 \%)$, had no prior connection in Geneva (74\%) and stayed in town less than 48 hours before being hospitalized (63\%).

Patients on a pathological journey were frequently admitted to the psychiatric hospital after police intervention (54\%) and/or transfer from the emergency services situated in the general hospital $(50 \%)$. Persecutory delusions were present in $53 \%$ of the sample, whereas hetero-aggressive or autoaggressive behavior was reported among $17 \%$ and $12 \%$, respectively. Length of stay at the psychiatric hospital was usually short (4 days or less for $67 \%$ of patients). At discharge, $58 \%$ were diagnosed with psychotic disorders (schizophrenia $\mathrm{N}=12$, acute transient psychotic disorders $\mathrm{N}=15$, delusional disorders $\mathrm{N}=7$, schizoaffective disorder
$\mathrm{N}=3$, other psychotic disorders $\mathrm{N}=7$ ) and $22 \%$ with mood disorders (bipolar disorder $\mathrm{N}=13$, unipolar depression $\mathrm{N}=4$ ). Seventy-two percent were prescribed antipsychotic medication. After establishing contact with patient's family or physician $(67 \%)$, transfers were organized to other structures in Geneva $(43 \%)$ or hospital in residence place $(33 \%)$, whereas $20 \%$ of patients were voluntarily repatriated to their home countries.

Of the 76 patients hospitalized after a pathological journey, $16(21 \%)$ specifically reported being attracted to Geneva because of its international organizations, while 60 came for other reasons. The two sub-samples are compared in Table 2. Patients attracted by international organizations differed from the other travelers as follows. They more often came from abroad ( $88 \%$ versus $63 \%, \mathrm{P}=0.063)$. They less often had prior connections in Geneva (6\% versus $32 \%$, $\mathrm{P}=0.054)$. Police intervention was less frequent (19\% versus $63 \%, \mathrm{P}=0.002$ ) but admissions more often occurred through emergency facilities ( $81 \%$ versus $42 \%, \mathrm{P}=0.010)$. Conditions of discharge from the hospital also significantly differed $(\mathrm{P}=0.015)$, with a lower proportion of patients voluntarily repatriated $(0 \%$ versus $25 \%)$ or transferred to hospital in residence place (25\% versus $35 \%$ ). Prevalence of persecutory delusions was significantly higher among travelers attracted by the "international Geneva" ( $88 \%$ versus $43 \%$, $\mathrm{P}=0.002$ ).

Different organizations were the target of unwanted visits: the UN (United Nations), HCR (High Commissioner for Refugees), ICRC (International Committee of the Red Cross) and WHO (World Health Organization) being the most frequently visited. A few representative clinical vignettes are presented below.

1. Mr. A., aged 30, born in Liberia, was an asylum seeker living in Germany. At age 29, he was hospitalized in Germany because he felt threatened and persecuted. After discharge, he spent several weeks wandering around in England and Ireland. He came to Geneva to seek help at the High Commissioner for Refugees (HCR). A few days after his arrival in Geneva and several phone calls to the HCR, he went to the local general hospital emergency department, complaining of itching that he attributed to intentional poisoning. Hospitalized in psychiatry with a diagnosis of delusional disorder, he refused repatriation to Germany. He improved with antipsychotic medication and was discharged 6 days later.

2. Mr. B., aged 25, born in Algeria was French and lived in Paris. He was single and unemployed. He came to Geneva in order to meet several representatives at various international organizations, claiming that the French authorities wanted to eliminate him. After unsuccessfully trying to obtain appointments, he went to the local general hospital emergency department asking for political asylum. His psychiatric history revealed several previous psychiatric hospitalizations and antipsychotic treatments in France, where he had been diagnosed with schizophrenia. Transferred to psychiatry, he refused treatment and repatriation. In the absence of imminent danger, he was discharged 48 hours later. 
Table 1. Description of Patients Hospitalized During Pathological Journeys to Geneva (N=76)

\begin{tabular}{|c|c|c|c|}
\hline \multicolumn{2}{|l|}{ Characteristics } & \multirow{2}{*}{$\frac{\mathbf{N}}{}$} & \multirow{2}{*}{$\frac{\% *}{57.9}$} \\
\hline Gender & Male & & \\
\hline & Female & 32 & 42.1 \\
\hline Age & Mean (SD) & 40 & $(12)$ \\
\hline \multirow[t]{3}{*}{ Country of residence $(\mathrm{N}=73)$} & Switzerland & 23 & 31.5 \\
\hline & Other European country & 45 & 61.6 \\
\hline & Non European country & 5 & 6.8 \\
\hline Marital status (N=65) & Single & 43 & 66.2 \\
\hline \multirow[t]{3}{*}{ Psychiatric history } & Psychiatric history present $(\mathrm{N}=70)$ & 59 & 84.3 \\
\hline & History of antipsychotic medication $(\mathrm{N}=51)$ & 30 & 58.8 \\
\hline & Discontinued psychiatric treatment $(\mathrm{N}=65)$ & 41 & 63.1 \\
\hline \multirow[t]{4}{*}{ Journey to Geneva } & Patient traveled alone & 70 & 92.1 \\
\hline & Patient traveled to Geneva directly $(\mathrm{N}=55)$ & 34 & 61.8 \\
\hline & Absence of connection in Geneva ( $N=73)$ & 54 & 74.0 \\
\hline & Length of stay in Geneva $\leq 2$ days $(\mathrm{N}=59)$ & 37 & 62.7 \\
\hline \multirow[t]{7}{*}{ Admission to the hospital } & After police intervention & 41 & 53.9 \\
\hline & Through emergency services & 38 & 50.0 \\
\hline & Difficulties communicating with patient & 6 & 7.9 \\
\hline & Persecutory delusions $(\mathrm{N}=74)$ & 39 & 52.7 \\
\hline & Other delusions & 30 & 40.5 \\
\hline & Auto-aggressive behavior & 9 & 11.8 \\
\hline & Hetero-aggressive behavior & 13 & 17.1 \\
\hline Psychiatric diagnosis & Psychotic disorders (F2x.xx) & 44 & 57.9 \\
\hline \multirow[t]{5}{*}{ according to ICD-10 } & Mood disorders (F3x.xx) & 17 & 22.4 \\
\hline & Personality disorders (F60.x or F69) & 6 & 7.9 \\
\hline & Psychoactive substance use $(\mathrm{F} 1 \mathrm{x} \cdot \mathrm{xx})^{\S}$ & 4 & 5.3 \\
\hline & Other diagnosis & 4 & 5.3 \\
\hline & No diagnosis & 1 & 1.3 \\
\hline \multirow[t]{3}{*}{ Duration of hospital stay } & 1 - 2 days & 33 & 43.4 \\
\hline & 3 - 4 days & 18 & 23.7 \\
\hline & $>4$ days & 25 & 32.9 \\
\hline \multirow[t]{2}{*}{ Prescribed medication } & Antipsychotic drug & 55 & 72.4 \\
\hline & Benzodiazepine & 28 & 36.8 \\
\hline \multirow[t]{7}{*}{ Discharge from the hospital } & After contact with patient's family & 18 & 23.7 \\
\hline & After contact with patient's doctor & 26 & 34.2 \\
\hline & After contact with both family and doctor & 7 & 9.2 \\
\hline & Transfer to hospital in region of residence & 25 & 32.9 \\
\hline & Repatriation & 15 & 19.7 \\
\hline & Transfer to other structure in Geneva & 33 & 43.4 \\
\hline & Patient ran away from hospital & 3 & 3.9 \\
\hline
\end{tabular}

${ }^{8}$ As main diagnosis; 11 other patients had substance abuse associated with another psychiatric diagnosis. *Percentages calculated for N=76, unless indicated otherwise.

SD: standard deviation.

3. $\quad$ Mrs. C., aged 45, was a British subject living in Scotland. Divorced, she had a teenaged daughter who had left home during the previous year. Convinced that her daughter was manipulated by the Irish Republican Army (IRA), she asked for help at Amnesty International in England. Unsatisfied with their response, she came to Geneva, feeling that the International
Committee of the Red Cross (ICRC) might have relevant information. As she refused to leave ICRC offices, the psychiatrist on duty was called and had her admitted in psychiatry. Repatriation was quickly organized and the patient was transferred to her country of residence 24 hours later. Despite possible suspicion of delusional disorder, she was discharged 
with a temporary diagnosis of acute transient disorder in the absence of additional information.

4. Mrs. D., aged 50, was Italian. Treated in her country for a bipolar disorder, she had been taking lithium since the age of 30. After treatment interruption, the patient became euphoric and left her place of residence for Venice, where she stayed for a few weeks. Eventually, she flew from Venice to Geneva. Upon arrival, Mrs. D went to the UN headquarters, asking for a subsidy to support the creation of a new sales company. Later on the same day, she went to the general hospital, asking for the removal of her psychiatric diagnosis. A manic episode was diagnosed and the patient was transferred to psychiatry, where she was successfully treated with olanzapine and lithium. Bipolar I disorder was confirmed and she voluntarily went back to her country.

\section{DISCUSSION AND CONCLUSIONS}

Despite its marginal importance (less than $1 \%$ of admissions), pathological journey represents an identifiable cause of psychiatric hospitalizations in Geneva. Among these patients, $21 \%$ do specifically travel to Geneva in order to contact or visit international organizations headquarters.

As similar studies are lacking, it is difficult to compare our figures with data from the literature. In 1981, French authors reported having retrospectively analyzed 214 medical records of pathological travelers, but presented qualitative data only [11]. Based on their clinical descriptions, these authors proposed a new typology of pathological travel. They distinguished between the simple, secondary and pure pathological travel. In the "simple pathological travel" traveling obeys to delusional ideation without being integrated into a delusional system. In these cases, destination is of secondary importance (e.g., traveling to escape surveillance by secret services). By contrast, in the "secondary pathological travel" travel is integrated into a delusional system and destination plays a central role (e.g., in response to a delusional order to commit suicide in a specific location). In "pure pathological travel" delusional ideation or hallucinations are focused on the necessity to travel (e.g., hearing voices ordering the patient to take the subway several times a day). According to this typology, most of our cases of pathological journeys to the international Geneva would fit into the category of "secondary pathological travel". Other French authors published a retrospective study of 29 pathological journeys to the Bordeaux area, in the south west region of France [12]. Their methodology was fairly similar to ours, since medical records were identified on the basis of admission certificates to a 20-bed inpatient unit. Their results were also comparable with the present study: patients were in majority male (20/29), with a mean age of 33 years. Most patients $(22 / 29)$ presented with delusional symptoms, usually of the persecutory type. In their discussion, authors note that the travel destination is usually a non-random choice, since journeys retain some degree of organization.

From a psychopathological perspective, it is important to note that no single mental disorder can account for all pathological journeys. The clinical examples presented above illustrate this point. However, acute transient psychotic disorders, schizophrenia and delusional disorders are the most frequent diagnostic categories among this sample. Persecutory delusions seem to be the underlying cause of travel in most cases, while delusions of grandiosity account for the cases encountered during manic episodes. The four patients who had a main diagnosis of psychoactive substance use had traveled in a state of street drugs intoxication.

This retrospective study has several limitations. Firstly, patients' medical records were selected on the basis of the content of hospital admission certificates. This method might possibly have underestimated the true number of pathological travelers. Indeed, the admission certificate is a brief document, written prior to admission, usually in emergency conditions. Some patients might have gone undetected since pathological travel is not systematically investigated or mentioned at initial assessment. However, because of strict legal rules regarding compulsory hospitalizations in psychiatry in our geographic area, the context and motives of emergency interventions is usually mentioned on certificates. Secondly, as most hospital stays were short, detailed data regarding psychopathology were often missing. The reason for the pathological journey may not have been scrupulously recorded in all case files. Thirdly, 60 of 76 subjects traveled for nonspecific reasons such as accessibility or random choice. As there was no control group comprising patients identified in another city using similar methods, the importance of the nonspecific determinants of travel can't be fully assessed. Fourthly, it is probable that most individuals visiting international organizations with inadequate demands were not referred to psychiatry. Therefore, our study addresses only a subgroup of pathological travelers to Geneva. Fifthly, the association between pathological journeys and specific psychotic disorders should be investigated in prospective studies. Indeed, our results indicate that active delusional ideation (mainly of the persecution type) put patients at risk for pathological journeys but does not allow for reliable comparison between diagnostic categories (schizophrenia, acute delusional psychoses and delusional disorders).

In the context of globalization, international travel is on the increase. Of course, this may lead to a rise in pathological journeys as explained above, but traveling to an international city such as Geneva may also express help seeking behavior, for example because of human rights abuse in the subject's country of origin [13]. One should be careful not to stigmatize such behaviors, no matter whether this is done in an appropriate manner or not.

In order to improve the scientific quality of future prospective studies and to limit the risk of stigmatizing people crossing borders to seek help, such as refugees or asylum seekers, we believe that a more precise definition of pathological journeys should be used. On the basis of available data, we suggest restricting this framework to conditions in which psychotic symptoms precede displacement and govern it (for example as a response to persecutory delusions). This definition excludes syndromes where mental disturbance is the consequence of displacement (such as the Paris or New York syndromes described among Japanese tourists, or culture shock, mainly described among expatriates [14]). This definition would also exclude the case of persecuted or desperate individuals traveling without preparation to ask for help in ways judged as inadequate. 
Table 2. Comparison of Patients Whose Journey was Related or Not with the International Tradition of Geneva

\begin{tabular}{|c|c|c|c|c|c|c|}
\hline & & \multicolumn{2}{|c|}{ Related (N=16) } & \multicolumn{2}{|c|}{ Not related $(N=60)$} & \multirow[b]{2}{*}{$\mathbf{P}^{\S}$} \\
\hline \multicolumn{2}{|l|}{ Characteristics } & $\mathbf{n}$ & $\% *$ & $\mathbf{n}$ & $\% *$ & \\
\hline \multirow[t]{2}{*}{ Gender } & Male & 11 & 68.8 & 33 & 55.0 & N.S. \\
\hline & Female & 5 & 31.3 & 27 & 45.0 & \\
\hline Age & Mean (SD) & 42 & $(10)$ & 39 & (13) & N.S. \\
\hline \multirow[t]{3}{*}{ Country of residence $(\mathrm{N}=16$ s 57$)$} & Switzerland & 2 & 12.5 & 21 & 36.8 & 0.063 \\
\hline & Other European country & 14 & 87.5 & 31 & 54.4 & \\
\hline & Non European country & 0 & 0.0 & 5 & 8.8 & \\
\hline \multirow[t]{2}{*}{ Journey to Geneva } & Without connection in Geneva $(\mathrm{N}=16$ vs 57$)$ & 15 & 93.8 & 39 & 68.4 & 0.054 \\
\hline & With connection in Geneva & 1 & 6.3 & 18 & 31.6 & \\
\hline \multirow[t]{7}{*}{ Admission to the hospital } & With police intervention & 3 & 18.8 & 38 & 63.3 & 0.002 \\
\hline & Without police intervention & 13 & 81.3 & 22 & 36.7 & \\
\hline & Through emergency services & 13 & 81.3 & 25 & 41.7 & 0.010 \\
\hline & Through other services & 3 & 18.8 & 35 & 58.3 & \\
\hline & Persecution delusions ( $\mathrm{N}=16$ vs 58 ) & 14 & 87.5 & 25 & 43.1 & 0.002 \\
\hline & Other delusions & 1 & 6.3 & 29 & 50.0 & \\
\hline & No delusions & 1 & 6.3 & 4 & 6.9 & \\
\hline \multirow[t]{4}{*}{ Discharge from the hospital } & Transfer to hospital in region of residence & 4 & 25.0 & 21 & 35.0 & 0.015 \\
\hline & Repatriation & 0 & 0.0 & 15 & 25.0 & \\
\hline & Transfer to other structure in Geneva & 10 & 62.5 & 23 & 38.3 & \\
\hline & Patient ran away from hospital & 2 & 12.5 & 1 & 1.7 & \\
\hline
\end{tabular}

*Percentages calculated for $\mathrm{N}=16$ and 60 , unless indicated otherwise.

${ }^{\S}$ Fisher's exact test for proportions and Student's t-test for continuous variables (2-sided). SD: standard deviation.

Using this operational definition of pathological travels, it would be interesting to compare data involving various cities notable for their international organizations, such as Strasbourg, Brussels, La Haye, New York and Geneva. Whether these situations are a source of concern for international organizations medical services should also be investigated. In our study, none of the analyzed cases truly jeopardized the people working in the international organizations.

\section{ACKNOWLEDGEMENTS}

The authors thank Jane Michaelis Zurbuchen for her help with the English version of the text.

\section{REFERENCES}

[1] Hacking I. Les Alienes voyageurs: how fugue became a medical entity. Hist Psychiatry 1996; 7(27 pt 3): 425-49.

[2] Tannock C, Turner T. Psychiatric tourism is overloading London beds. BMJ 1995; 311(7008): 806

[3] American Psychiatric Association: Diagnostic and statistical manual of mental disorders, 4th ed. Washington (DC): APA; 1994.
[4] Word Health Organization: The ICD-10 Classification of Mental and Behavioural Disorders: Clinical Descriptions and Diagnostic Guidelines: WHO; 1992

[5] Caro F. Pathological displacement: background and differential diagnoses. L'Information Psychiatrique 2006; 82: 405-415.

[6] Fastovsky N, Teitelbaum A, Zislin J, Katz G, Durst R. The Jerusalem syndrome. Psychiatr Serv 2000; 51(8): 1052.

[7] Kalian M, Witztum E. Comments on Jerusalem syndrome. Br J Psychiatry 2000, 176: 492 .

[8] Fastovsky N, Teitelbaum A, Zislin J, Katz G, Durst R. Jerusalem syndrome or paranoid schizophrenia? Psychiatr Serv 2000; 51(11): 1454

[9] Kalian M, Witztum E. Jerusalem syndrome or paranoid schizophrenia? Psychiatr Serv 2000; 51(11): 1453-4.

[10] Airault R. Les fous de l'Inde. Vol 1. Paris: Payot; 2000

[11] Caroli F, Masse G. [The concept of a pathologic trip]. Ann Med Psychol (Paris) 1981; 139(7): 828-32.

[12] Verdoux H, Goumilloux R, Bourgeois M: Trips and psychiatric pathology: a sample of 29 cases. Annales médico-psychologiques 1993; 151: 581-5.

[13] Kelly B. Globalisation and psychiatry. Advances in Psychiatric Treatment 2003; 9: 464-74.

[14] Stewart L, Leggat PA. Culture shock and travelers. J Travel Med 1998; 5(2): 84-8 